\title{
Preensão palmar e cognição em idosas institucionalizadas e residentes na comunidade
}

\author{
Handgrip strength and cognition in community and institution living elderly women
}

\author{
Cristiane Scodeler Crispim ${ }^{1}$, Thais de Lima Resende ${ }^{2}$
}

\begin{abstract}
RESUMO
Objetivo: Avaliar a força de preensão palmar (FPP) e a cognição em idosas institucionalizadas e residentes na comunidade, verificar se existe uma associação entre cognição e FPP nessa população e comparar os dois grupos.

Materiais e Métodos: Em uma amostra por conveniência, participaram 22 idosas residentes em uma instituição (GI) de Porto Alegre/RS e 27 residentes na comunidade (GC), membros de um grupo de idosos em Gravataí/RS. Foram avaliadas a cognição (Mini-exame do Estado Mental - MEEM) e a FPP (dinamômetro Jamar®). Também foram coletados dados sociodemográficos, de saúde, entre eles o índice de massa corporal (IMC), sobre dominância, apresentação física e prática de atividade física.

Resultados: A média de idade das idosas do Gl foi $78,6 \pm 7,5$ anos e do GC foi $74,7 \pm 6,9$ anos. As voluntárias eram destras (100\%), da raça branca $(83,7 \%)$, com 1 a 8 anos de estudo $(91,8 \%)$, deambulavam sem auxílio (82\%), não praticavam atividade física $(67,3 \%)$, com IMC igualmente dividido entre normal $(40,8 \%)$ e acima do peso $(40,8 \%)$. Gl e GC não diferiram em termos de FPM, mas as idosas da comunidade apresentaram escores do MEEM significativamente mais altos. Idade apresentou uma correlação negativa, moderada e significativa $(r=-0,52 ; p<0,01)$ com o escore do MEEM e negativa de grau fraco com a FPM ( $r=-0,28 ; p=0,05)$. Não foi detectada relação entre a FPP e o IMC.

Conclusão: Idosas institucionalizadas apresentaram nível cognitivo inferior às da comunidade, porém não diferiram delas em termos de força. FPP e cognição diminuem com o aumento da idade.
\end{abstract}

Palavras-chave: idoso; saúde do idoso; saúde do idoso institucionalizado; força muscular; cognição.

\section{ABSTRACT}

Objective: To assess hand grip strength (HGS) and cognition in women residing in the community and in a long-term institution for the elderly, to determine if there is an association between cognition and HGS in this population and to compare the two groups.

Materials and Methods: From a convenience sample, took part in this study 22 women from an institution in Porto Alegre/RS (IG) and 27 residents in the community (CG), members of an elderly group from Gravataí/RS. Their cognition (Mini-Mental State Examination - MMSE) and HGS (Jamar \& dynamometer) were assessed. The following data were also collected: sociodemographic, health, body mass index (BMI), hand dominance, physical presentation and physical activity.

Results: The mean ages were $78.6 \pm 7.5$ years for the $I G$ and $74.7 \pm 6.9$ for the $C G$. The women were right-handed (100\%), caucasian (83.7\%), with 1-8 years of education (91.8\%), ambulating without assistance (82\%), physically inactive (67. 3\%), with BMI equally divided between normal (40.8\%) and overweight (40.8\%). IG and CG did not differ in terms of HGS, but the community elderly had MMSE scores significantly higher. Age showed a negative, moderate and significant relationship $(r=-0.52, p<0.01)$ with the MMSE score and a negative, weak but significant relationship with HGS $(r=-0.28, p=0.05)$. No relation was detected between the HGS and BMI.

Conclusion: Institutionalized women had lower cognitive level than their community counterparts, but did not differ in terms of strength. HGS and cognition decline with increasing age.

Keywords: aged; health of the elderly; health of institutionalized elderly; muscle strength; cognition.

\footnotetext{
${ }^{1}$ Fisioterapeuta. Especialista em Acupuntura (CBES).

${ }^{2}$ Fisioterapeuta. Doutor em Ciências da Saúde (PUCRS). Professora Titular da Faculdade de Enfermagem, Nutrição e Fisioterapia da Pontifícia Universidade Católica do Rio Grande do Sul.
} 


\section{INTRODUÇÃO}

A população idosa se caracteriza por apresentar mais fatores de risco para doenças crônicodegenerativas ${ }^{1}, 0$ que, em associação com crescimento exponencial da população idosa e o crescente aumento da perspectiva de vida, leva ao aumento do número de pessoas portadoras de incapacidades que requerem atenção direta e cuidados intensivos, sobrecarregando o sistema de saúde, que ainda não está apto a receber este novo contingente populacional ${ }^{2}$.

A modificação do perfil epidemiológico da população brasileira evidencia sobremaneira a necessidade de se mudar efetivamente a direção da atenção à saúde da população idosa, transferindo o seu foco para a promoção da saúde ${ }^{2}$. Assim, segundo Fonseca e Rizzoto $^{3}$, para que se consiga um envelhecimento saudável e independente são necessárias tecnologias que permitam realizar diagnósticos corretos da situação de cada idoso, para então se traçar metas e intervir de uma maneira mais adequada. Deste modo, é comum o uso combinado de vários instrumentos de avaliação, formando protocolos de avaliação e tratamento diversos para múltiplos acometimentos ${ }^{4}$.

O processo de envelhecimento humano leva a várias modificações, entre elas a diminuição e lentidão gradual de movimentos resultante de uma combinação de perda de fibras musculares e, consequentemente, perda de força muscular, e também da desnervação das fibras musculares e do comprometimento da placa motora ${ }^{1,5}$. Assim, as dificuldades na realização de tarefas que necessitam de coordenação motora fina são sinais que podem demonstrar a perda de força muscular ${ }^{6}$.

Boa parte dos estudos atuais a respeito da função manual tende a se concentrar na força. Provavelmente isto ocorre porque a avaliação da força é um dos aspectos da função manual mais fácil de se medir de uma forma prática e objetiva. Além disso, a força de preensão manual de idosos apresenta uma relação diretamente proporcional ao quadro de força muscular geral. Afora essa relação, a força de preensão manual é essencial para a realização das atividades de vida diária6 e importante fator de predição da redução da capacidade funcional e mobilidade ${ }^{7}$ e da qualidade de vida em idosos ${ }^{8}$. A sua mensuração e o seu acompanhamento longitudinal justificam-se na medida em que se sabe que ela sofre uma perda média de $2 \%$ ao ano em idosos, perda essa que se acelera com o avançar da idade, principalmente nas mulheres ${ }^{9}$.

No entanto, cabe ressaltar que, para uma boa qualidade de vida o idoso necessita contar com a manutenção da capacidade cognitiva, além de força e condicionamento. É importante ressaltar que a prevalência da demência dobra a cada cinco anos de aumento da idade, indo de $3 \%$ aos 70 anos para 20 $30 \%$ aos 85 anos $^{10}$.

O perfil cognitivo de uma população idosa pode ser obtido através dos resultados de testes cognitivos padronizados. Entre os muitos instrumentos utilizados para esse fim, pode-se citar o "Mini-exame do Estado Mental" (MEEM) de Folstein et al. ${ }^{11}$, que tem sido amplamente utilizado na pesquisa e na clínica do indivíduo idoso e serve para um rastreamento inicial do estado mental ${ }^{12}$.

Estratégias de mudança do perfil de dependência do idoso têm sido pensadas e elaboradas ao longo dos anos com a finalidade de se obter melhora da qualidade do viver mais. A manutenção da capacidade funcional dos idosos, dentro da individualidade e da especificidade de cada situação, constitui um dos papéis mais importantes dos profissionais de saúde. A avaliação funcional dos idosos torna-se, então, essencial para estabelecer um bom diagnóstico, um prognóstico e um julgamento clínico adequado, servindo de base para as decisões sobre os tratamentos e cuidados necessários ${ }^{4,13}$.

Deste modo, o presente estudo teve como objetivos avaliar a força muscular de preensão palmar e o nível de cognição em idosas que residem na comunidade e em idosas institucionalizadas, verificar se nessa população existe uma associação entre a função cognitiva e a força de preensão palmar e comparar os dois grupos de idosas em termos das variáveis medidas.

\section{MATERIAIS E MÉTODOS}

Trata-se de um estudo observacional, analítico e transversal, realizado na instituição asilar Lar Maria de Nazaré, no município de Porto Alegre (RS) e em uma comunidade do município de Gravataí (RS), no período 
de maio de 2008, após aprovação do Comitê de Ética e Pesquisa (CEP) da Universidade do Vale do Rio dos Sinos (resolução: 013/2008; $n^{\circ}$ projeto: CEP 08/015).

Nessa amostra por conveniência, foram incluídas as mulheres com mais de 60 anos de idade, que aceitaram participar do projeto. As participantes foram divididas em dois grupos, de acordo com o seu local de residência, o Grupo Comunidade e o Grupo Instituição.

Foram excluídas do estudo as mulheres: com idade inferior a 60 anos, que não compreenderam as informações fornecidas pela pesquisadora durante a coleta de dados, com doenças ou sequelas de doenças neurológicas, reumáticas e/ou osteomusculares que pudessem interferir direta ou indiretamente no desempenho dos testes aplicados e aquelas que não demonstraram interesse em participar do estudo.

Os dados foram coletados em formulário próprio, após terem sido explicados às participantes os objetivos e procedimentos do estudo e após as mesmas terem assinado o Termo de Consentimento Livre e Esclarecido. Todas as medidas e testes foram realizados pela mesma pesquisadora. Foram coletados dados pessoais (idade, raça, escolaridade, dominância), dados relativos à apresentação física, realização de atividade física e presença de alguma doença que pudesse alterar a confiabilidade dos dados, assim como foram mensuradas a estatura, a massa corporal, a preensão palmar e foi avaliada a função cognitiva.

A estatura foi mensurada através de uma fita métrica. A postura padrão recomenda ângulo reto procurando colocar os calcanhares, a cintura pélvica, a cintura escapular e a região occipital na posição correta. Para aferição foi utilizado um esquadro de madeira colocado rente à cabeça fazendo um ângulo de 90․ A massa corporal foi registrada em quilogramas e obtida através de uma balança digital, aferida para o estudo e colocada em local plano (usado um nível). As mulheres sempre foram avaliadas usando roupas leves. Foi solicitado àquelas que estavam vestindo roupas de frio que as retirassem, antes que fosse efetuada a medida. A partir dos dados da estatura e da massa corporal foi calculado o índice de massa corporal (IMC), o qual foi utilizado para ordenar as voluntárias quatro categorias: (1) abaixo do peso, (2) peso normal, (3) sobrepeso e (4) obesa ${ }^{14}$.
A força de preensão palmar manual das idosas foi avaliada com um dinamômetro da marca Jamar® segundo as recomendações da Sociedade Americana de Terapeutas de Mão (SATM) ${ }^{15}$. Após a adoção do posicionamento correto, foram realizadas três medidas intercaladas da mão direita (MD) e também da mão esquerda (ME). Considerou-se a maior entre as três medidas em kilogramas-força (Kgf) para o resultado efetivo do teste, conforme recomenda a $\operatorname{SATM}^{14}$ no protocolo de utilização do dinamômetro Jamar®.

A avaliação da função cognitiva foi feita através do Mini-Exame do Estado Mental (MEEM), elaborado por Folstein et al. ${ }^{11}$ e validado para população brasileira por Bertolucci et al. ${ }^{12}$. O MEEM é um teste cognitivo breve, composto por itens a respeito de orientação temporoespacial, registro, memória de curto prazo, atenção, cálculo, linguagem e praxia construcional, possibilitando uma avaliação do estado mental de um paciente. Segundo Bertolucci et al. ${ }^{12}$, o ponto de corte para analfabetos é de 13 pontos, 18 pontos para 1 a 7 anos de estudo e 26 pontos para 8 anos ou mais. Esses pontos de corte foram adotados no presente estudo.

A análise dos dados teve como abordagem inicial a estatística descritiva com a distribuição de frequências simples e relativa, bem como, as medidas de tendência central (média e mediana) e de variabilidade (desvio padrão e amplitude interquartílica). Também foi realizada a análise exploratória das variáveis quantitativas objetivando um maior conhecimento sobre a distribuição dessas, através do teste de KolmogorovSmirnov, quando foi avaliado o total da amostra, e pelo teste de Shappiro-Wilk, quando a avaliação foi feita por grupos. Na comparação dos escores entre os dois grupos independentes, foi implementado o teste de Mann-Whitney, em função de que algumas variáveis não apresentaram uma distribuição aproximadamente normal. Para a investigação de possível associação entre variáveis quantitativas foi utilizado o Coeficiente de Correlação de Pearson e nas situações onde a distribuição de pelo menos uma das variáveis não era aproximadamente normal, foi implementado o coeficiente de correlação de Spearman.

Os dados receberam tratamento estatístico utilizandose o software SPSS 11.5 (Statistical Package to Social 
Sciences for Windows) onde, para critérios de decisão foi adotado o nível de significância $(\alpha)$ de $5 \%$ e, para significância limítrofe os níveis de significância compreendidos entre 5 e 10\%. A análise dos dados foi realizada sob cegamento, por uma pessoa que desconhecia o projeto, bem como o significado das variáveis.

\section{RESULTADOS}

Participaram do estudo 53 idosas, sendo quatro excluídas por apresentar disfunções em membros superiores que comprometeriam o resultado do teste de força. Deste modo, a amostra final foi composta por 49 voluntárias (61-92 anos; 76,5 $\pm 7,5$ anos), sendo 22 idosas institucionalizadas (44,4\%) com média de idade $78,6 \pm 7,5$ anos, e 27 idosas da comunidade (55,6\%) com média de idade $74,7 \pm 6,9$ anos. Todas as voluntárias eram destras.

Como pode ser visto na tabela 1, a maior parte era da raça branca $(83,7 \%)$, com a distribuição percentual equivalente entre os dois grupos. $O$ grau de escolaridade de $91,8 \%$ das idosas ficou entre 1 a 8 anos de estudo, no entanto foi encontrado um maior número de idosas com ensino médio completo na comunidade. Em relação ao IMC, 20 idosas (40,8\%) apresentaram valores considerados normais; a mesma proporção foi encontrada para quem estava acima do peso (40,8\%). Apenas oito mulheres foram consideradas obesas $(16,4 \%)$, sendo a maioria delas encontradas no Grupo da Comunidade. Apenas $2 \%$ das mulheres estavam abaixo do peso, todas elas institucionalizadas (tabela 1). Não foi encontrada correlação entre o IMC e a força de preensão manual nas idosas avaliadas, tanto na amostra total, quanto nos dois grupos separadamente.

A maioria das idosas não praticava atividade física $(67,3 \%)$. Dentre as 16 mulheres que praticavam atividade física regularmente, 15 delas vivem na comunidade (tabela 1). De acordo com a apresentação, $82 \%$ delas deambulavam sem auxílio, e apenas uma idosa da instituição encontrava-se acamada. Não foi encontrada correlação significativa entre a apresentação e a força de preensão palmar, deste modo não houve diferença entre as idosas que deambulavam com auxílio e as que não.

TABELA 1 - Características das idosas por local de residência.

\begin{tabular}{|c|c|c|c|c|}
\hline Características* & & Total & Grupo Instituição & Grupo Comunidade \\
\hline \multirow{3}{*}{ Raça } & Brancos & $41(83,7)$ & $18(81,8)$ & $23(85,2)$ \\
\hline & Negros & $3(6,1)$ & $2(9,1)$ & $1(3,7)$ \\
\hline & Pardos & $5(10,2)$ & $2(9,1)$ & $3(11,1)$ \\
\hline \multirow{4}{*}{ Escolaridade } & Analfabetos & $3(6,1)$ & $2(9,0)$ & $1(3,7)$ \\
\hline & 1 a 4 anos & $23(46,9)$ & $14(63,7)$ & $9(33,3)$ \\
\hline & 5 a 8 anos & $22(44,9)$ & $5(22,8)$ & $17(63,0)$ \\
\hline & 9 ou mais & $1(2,1)$ & $1(4,5)$ & $0(0,0)$ \\
\hline \multirow{4}{*}{ IMC } & Abaixo do peso & $1(2,0)$ & $1(4,5)$ & $0(0,0)$ \\
\hline & Normal & $20(40,8)$ & $12(54,6)$ & $8(29,6)$ \\
\hline & Sobrepeso & $20(40,8)$ & $7(31,9)$ & $13(48,2)$ \\
\hline & Obeso & $8(16,4)$ & $2(9,0)$ & $6(22,2)$ \\
\hline \multirow{2}{*}{ Atividade Física } & Não & $33(67,3)$ & $21(95,5)$ & $12(44,4)$ \\
\hline & Sim & $16(32,7)$ & $1(4,5)$ & $15(55,6)$ \\
\hline \multirow{3}{*}{ Apresentação } & Acamado & $1(2,0)$ & $1(4,5)$ & $0(0,0)$ \\
\hline & Deambula com auxílio & $8(16,0)$ & $2(9,1)$ & $6(22,2)$ \\
\hline & Deambula sem auxílio & $40(82,0)$ & $19(86,4)$ & $21(77,8)$ \\
\hline
\end{tabular}

*Dados apresentados na forma de n (\%); n: tamanho amostral; IMC: Índice de Massa Corporal. 
TABELA 2 - Comparação das idosas em termos de força e cognição.

\begin{tabular}{lccccc}
\hline Variável & & Total $(\mathrm{n}=49)$ & Grupo Instituição $(\mathrm{n}=22)$ & Grupo Comunidade $(\mathrm{n}=27)$ & $\boldsymbol{p}^{*}$ \\
\hline \multirow{2}{*}{ FPP (Kgf) } & Mediana & 20 & 18 & 22 &, 122 \\
& $(\mathrm{Q} 1-\mathrm{Q} 3)$ & $(16-24)$ & $(14-24)$ & $(18-26)$ & 29 \\
MEEM & Mediana & 26 & 23 & $(26-30)$ & $<, 001$
\end{tabular}

n: tamanho amostral; p: probabilidade; *: teste de Mann-Whitney; FPP: força de preensão palmar; Kgf: quilograma-força; Q1-Q3: intervalo interquartil; MEEM: Mini Exame do Estado Mental.

Conforme pode ser visto na tabela 2 , a média de força para o total da amostra foi de $20 \pm 6,1 \mathrm{Kgf}$. Apesar das idosas institucionalizadas $(18,7 \pm 5,9 \mathrm{Kgf})$ apresentarem FPP inferior às idosas da comunidade (21 $\pm 5,9 \mathrm{Kgf})$, essa diferença não foi significativa $(p=0,12)$.

Em termos de escore do MEEM (tabela 2), as idosas da comunidade apresentaram mediana de 29 pontos, enquanto que as idosas institucionalizadas apresentaram mediana de 23 pontos. A diferença entre os grupos se mostrou significativa $(p>0,01)$. Apenas quatro voluntárias (8\%) apresentaram escore do MEEM compatível com possível demência, todas elas institucionalizadas e todas com escolaridade entre 4 e 8 anos. As variáveis idade e escore do MEEM apresentaram uma correlação negativa, moderada e significativa $(r=-0,52 ; p<0,01)$. Essa correlação é ainda mais forte no grupo institucionalizado (tabela 3 ).

Considerando a idade das voluntárias e os resultados de força obtidos, foi detectada correlação estatisticamente significativa entre essas variáveis, onde o coeficiente se mostrou negativo e de grau fraco para o total da amostra $(r=-0,28 ; p=0,05)$ indicando que, quanto maior a idade, menor a força. Esta relação negativa está presente nas idosas da comunidade $(r=-0,25 ; p=0,05)$, enquanto que no grupo de idosas asiladas esta correlação não foi detectada $(r=-0,25 ; p=0,65)$ (tabela 3).

Com a finalidade de verificar a relação entre a força de preensão palmar e o resultado do MEEM, foram calculados os coeficientes de correlação para toda a amostra e para os grupos. Foi observada uma correlação moderada e significativa entre as variáveis, tanto na amostra total, quanto nos grupos separadamente (tabela 3).

\section{DISCUSSÃo}

Foi encontrada uma associação entre a força de preensão palmar e a cognição, tanto na amostra total, quanto nos grupos separadamente, confirmando achados de outros estudos relativos à associação entre essas variáveis ${ }^{16-18}$. Esses resultados foram obtidos tanto em estudos transversais ${ }^{18}$, quanto em estudos longitudinais ${ }^{16,17}$. Anstey et al. $^{18}$ relataram que marcadores não cognitivos, como força de preensão palmar, explicaram as diferenças de desempenho cognitivo em 180 mulheres com idades entre os 60 a 90 , resultado corroborado pelo estudo longitudinal com duração de sete anos de Alfaro-Acha et al. $^{16}$, onde os idosos que apresentaram o menor resultado no teste de preensão palmar apresentaram também o maior déficit cognitivo.

A natureza da associação entre força muscular e cognição permanece incerta, mas as medidas de força podem ser vistas como um indicador geral da integridade do sistema nervoso central, por também serem sensíveis ao processo de envelhecimento ${ }^{19,20}$. Vale dizer que essa associação não se resume à força muscular e função cognitiva. Outros marcadores do desempenho físico (marcha, velocidade e equilíbrio), assim como marcadores biológicos do processo de envelhecimento (estresse oxidativo, marcadores inflamatórios e marcadores hormonais) também têm apresentado uma associação com a função cognitiva e/ou muscular ${ }^{21}$.

Rosano et al. $^{22}$ encontraram uma correlação significativa entre os baixos desempenhos físicos (marcha, velocidade, equilíbrio e força) com pior desempenho na função cognitiva em 2893 idosos. Segundo os autores, outra explicação para a associação 
entre a baixa força muscular e o déficit cognitivo é a presença de alguns fatores como o estresse oxidativo, marcadores inflamatórios e marcadores hormonais em baixos níveis que possam contribuir para perda muscular e declínio cognitivo, todos eles indicativos do processo de envelhecimento.

Weaver et al. $^{23}$ demonstraram uma associação significativa entre elevação de marcadores inflamatórios no plasma e o risco para posterior declínio cognitivo em 779 idosos durante 2,5 anos de acompanhamento. Em outra pesquisa realizada, Cesari et al. ${ }^{24}$ descreveram uma relação inversa entre níveis elevados de marcadores inflamatórios e menor desempenho de força manual em 1.020 idosos. Estes achados sugerem a presença de um mecanismo comum entre o declínio cognitivo, perda de força muscular e declínio biológico com a idade.

Investigações recentes têm-se concentrado na identificação de fatores de risco que são prognósticos de futuro declínio cognitivo, visto que, ao identificar um fator de risco será possível atuar precocemente para se tentar prevenir a instalação de quadros demenciais e a consequente dependência do indivíduo por eles acometido. Alternativamente, se não for possível parar o processo de declínio cognitivo, pode-se tentar diminuir a velocidade da evolução dessa perda. Isso é particularmente importante frente aos gastos gerados pela dependência física, assim como pelo fato de que há décadas se sabe que o exercício físico resulta em fluxo cerebral sanguíneo maior e melhor função $\operatorname{cognitiva}^{25}$, melhor tempo de reação simples e de reação de escolha ${ }^{26}$, além das esperadas e conhecidas vantagens do mesmo para os idosos: maior mobilidade $^{27}$ e níveis maiores de independência funcional ${ }^{28}$.

Outro aspecto relevante é a relação da força com diferentes raças. Rantanen et al. ${ }^{29}$, em estudo realizado com 919 indivíduos, demonstraram que a força muscular difere em mulheres idosas por raça. No presente estudo não foi encontrada associação significativa entre as variáveis força e raça, achado esse que talvez seja explicado pelo fato de que, além de pequena, a amostra ter sido composta predominantemente por mulheres brancas.
A relação da força com a idade também tem sido relatada com frequência ${ }^{30-32}$. A força de preensão é diretamente proporcional à idade até os 30 anos aproximadamente, quando tem início uma redução gradual e progressiva da força, tornando-se clinicamente mais perceptível a partir dos 60 anos. No presente estudo esta relação também foi encontrada, porém com grau de correlação fraco, que talvez possa ser explicado pela pequena variação de idade na amostra (61-92 anos) e pelo pequeno tamanho amostral em relação aos outros estudos que analisaram amostras muito maiores de indivíduos em diferentes faixas etárias.

Apenas quatro voluntárias apresentaram resultados para o MEEM abaixo dos pontos de corte estabelecidos por Bertolucci et al. ${ }^{12}$ para população brasileira. Todas eram institucionalizadas e mais velhas, sendo que nenhuma delas era analfabeta. Com a institucionalização, o idoso passa a ser mais vulnerável ao declínio de sua função cognitiva através da dependência promovida pelos cuidadores, sendo a falta de estímulo uma das principais causas da aceleração desse declínio ${ }^{33}$.

Não foi encontrada diferença significativa de força entre as voluntárias que praticam atividade física, com uma frequência de no mínimo três vezes na semana. Programas de exercícios para idosos podem levar à melhora tanto da força física como da função cognitiva segundo alguns autores ${ }^{34}$. Esta é uma área importante para a futura investigação experimental.

Alguns trabalhos sobre a força de preensão palmar procuram correlacionar essa força ao índice de massa corporal, porém os resultados encontrados não são conclusivos $^{30,35}$. No presente estudo força de preensão palmar e IMC não se correlacionaram, enquanto Hulens et al. $^{35}$ observaram que a força era de $10-16 \%$ menor nas obesas em relação às não-obesas e Jürimäe e Jürimäe ${ }^{36}$ relataram maior força em mulheres acima do peso. Deste modo, a relação entre IMC e força de preensão palmar ainda não foi esclarecida.

Sabe-se que o bom funcionamento cognitivo é de grande importância para o êxito do envelhecimento e uma vida independente e autônoma. Deste modo, a identificação precoce de fatores que precedem o 
TABELA 3 - Relação entre idade, cognição e força, para o total da amostra, o Grupo Instituição e o Grupo Comunidade.

\begin{tabular}{lcccc}
\hline Correlação & & Total & Grupo Instituição & Grupo Comunidade \\
\hline Idade x MEEM & $r^{*}$ &,- 52 &,- 54 &,- 40 \\
& $\mathrm{p}$ & $<, 01$ &, 01 &, 04 \\
Idade x FPP & $r^{\dagger}$ &,- 28 &,- 25 &,- 30 \\
& $\mathrm{p}$ &, 05 &, 07 &, 05 \\
MEEM x Força & $r^{\phi}$ &, 56 &, 58 &, 42 \\
& $\mathrm{p}$ & $<, 01$ & $<, 01$ &, 03 \\
\hline
\end{tabular}

MEEM: escore do Mini-exame do Estado Mental; FPP: força de preensão palmar; $r^{\star}$ : Coeficiente de Correlação de Spearman; $\mathrm{p}$ : probabilidade; $r^{*}$ : Coeficiente de Correlação de Pearson.

declínio cognitivo é de importante implicação prática, especialmente se esses fatores são modificáveis. Um programa de exercício que melhora a força muscular poderia ajudar a prevenir ou retardar o declínio cognitivo em idosos, especialmente naqueles com força muscular reduzida.

Em conclusão, no presente estudo as idosas institucionalizadas apresentaram nível cognitivo inferior às da comunidade, porém não diferiram delas em termos de força, a qual, assim como a cognição, diminui com o aumento da idade.

\section{REFERÊNCIAS}

1. Troen RB. The biology of aging. Mt Sinai $\mathrm{J}$ Med. 2003;70(1):3-22.

2. Schramm JMA, Oliveira AF, Leite IC, Valente JC, Gadelha AMJ, Portela MC, et al. Transição epidemiológica e o estudo de carga de doença no Brasil. Ciênc Saúde Coletiva. 2004;9(4):897-908.

3. Fonseca FB, Rizzotto MLF. Construção de instrumento para avaliação sócio-funcional em idosos. Texto Contexto Enferm 2008;17(2):365-73.

4. den Ouden ME, Schuurmans MJ, Arts IE, van der Schouw YT. Physical performance characteristics related to disability in older persons: a systematic review. Maturitas. 2011;69(3):208-19.

5. Goodpaster BH, Park SW, Harris, Kritchevsky SB, Nevitt $\mathrm{M}$, Schwartz AV, et al. The loss of skeletal muscle strength, mass, and quality in older adults: the health, aging and body composition study. J Gerontol A Biol Sci Med Sci. 2006;61(10):1059-64.

6. Bohannon RW. Hand-grip dynamometry predicts future outcomes in aging adults. J Geriatr Phys Ther. 2008;31(1):3-10.

7. Lauretani F, Russo CR, Bandinelli S, Bartali B, Cavazzini $\mathrm{C}$, et al. Age-associated changes in skeletal muscles and their effect on mobility: an operational diagnosis of sarcopenia. J Appl Physiol. 2003;95(5):1851-60.

8. Sayer AA. Is grip strength associated with health-related quality of life? Findings from the Hertfordshire Cohort Study. Age Ageing 2006;35(4):409-15.
9. Frontera WR, Hughes VA, Lutz KJ, Evans WJ. A cross sectional study of muscle strength and mass in 45- to 78yr-old men and women. J Appl Phys.1991;71(2):644-50.

10. Scazufca M, Menezes PR, Almeida OP. Caregiver burden in an elderly population with depression in São Paulo, Brazil. Soc Psychiatry Psychiatr Epidemiol. 2002;37(9):416-22.

11. Folstein MF, Folstein SE, Mchugh PR. Mini-mental state: a practical method for grading the cognitive state of patients for the clinician. J Psychiatr Res 1975;12(3):189-98.

12. Bertollucci PHF, Brucci SMD, Campacci SR et al. O miniexame do estado mental em uma população geral: o impacto da escolaridade. Arq Neuro-psiquiatr 1994;52(1):1-7.

13. Ellis G, Whitehead MA, Robinson D, O'Neill D, Langhorne $P$. Comprehensive geriatric assessment for older adults admitted to hospital: meta-analysis of randomised controlled trials. BMJ. 2011;343:d6553. doi: 10.1136/bmj.d6553.

14. World Health Organization. Obesity: preventing and managing the global epidemic: report of a WHO consultation. Geneva: WHO; 2000. Technical Report Series 894. $265 \mathrm{p}$.

15. Figueiredo IM, Sampaio RF, Mancini MC, Silva FCM, Souza MAP. Teste da força de preensão utilizando o dinamômetro Jamar. Acta Fisiatr 2007;4(2):104-10.

16. Alfaro-Acha A, Al Snih S, Raji MA. Handgrip strength and cognitive decline in older mexican americans. J Gerontol A Biol Sci Med Sci. 2006;61(8):859-65.

17. Raji MA, Kuo YF, Snih SA, Markides KS, Peek MK, Ottenbacher KJ. Cognitive status, muscle strength, and subsequent disability in older Mexican Americans. J Am Geriatr Soc. 2005;53(9):1462-8.

18. Anstey KJ, Smith GA. Interrelationship among biological markers of aging, health, activity, acculturation, and cognitive performance in late adulthood. Psychol Aging 1999;14:605-18.

19. Christensen H, Mackinnon AJ, Korten A, Jorn AK. The "common cause hypothesis" of cognitive aging: evidence for not only a common factor but also specific associations of age with vision and grip strength in a cross-sectional analysis. Psychol Aging 2001;16(4):588-99.

20. Albert MS, Jones K, Savage CR, Berkman L, Seeman T, Blazer D, et al. Predictors of cognitive change in older persons: Mac Arthur Studies of Successful Aging. Psychol Aging 1995;10(4):578-89.

21. Szule P, Duboeuf F, Marchand F, Delmas PD. Hormonal and lifestyle determinants of appendicular skeletal muscle mass in men: The MINOS study. Am J Clin Nutr. 2004;80:496-503. 
22. Rosano C, Simonsick EM, Harris TB, Kritchevsky SB, Brach J, Visser M, et al. Association between physical and cognitive function in healthy elderly: The health, aging and body composition study. Neuroepidemiology 2005; 24(12):8-14.

23. Weaver JD, Huang $\mathrm{MH}$, Albert M, Harris T, Rowe JW, Seeman TE. Interleukin-6 and risk of cognitive decline: MacArthur Studies of Successful aging. Neurology 2002;59(3):371-8.

24. Cesari M, Penninx BW, Pahor M, Lauretani F, Corsi AM, et al. Inflammatory markers and physical performance in older persons: the InCHIANTI study. J Gerontol Med Sci 2004;59(3):242-8.

25. Rogers RL, Meyer JS, Mortel KF. After reaching retirement age physical activity sustains cerebral perfusion and cognition. J Am Geriatr Soc. 1990;38(2):123-8.

26. Rikli RE, Busch S. Motor performance of women as a function of age and physical activity level. J Gerontol 1986;41(5):645-9.

27. Lacroix AZ, Guralnik JM, Berkman LF, Wallace RB, Satterfield S. Maintaining mobility in late life: II. smoking, alcohol consumption, physical activity, and body mass index. Am J Epidemiol. 1993;137(8):858-69.

28. Mor V, Murphy J, Masterson-Allen S, Willey C, Razmpour $A$, Jackson ME, et al. Risk of functional decline among well elders. J Clin Epidemiol. 1989;42(9):895-904.

29. Rantanen T, Guralnik JM, Leveille S, Izmirlian G, Hirsch R, Simonsik E, et al. Racial differences in muscle strength in disabled older women. J Gerontol A Biol Sci Med Sci 1998;53(5):B355-61.

30. Mathiowetz V, Kashman N, Volland G, Weber K, Dowe M, Rogers S. Grip and pinch strength: normative data for adults. Arch Phys Med Rehabil. 1985;66(2):69-74.

31. Kaya A, Ozgocmen S, Ozge A, Kamanlin A, Gudul H. Relationship between grip strength and hand bone mineral density in healthy adults. Arch Med Res. 2005;36(5):603-6.

32. Stenholm S, Tiainen $\mathrm{K}$, Rantanen $\mathrm{T}$, Sainio $\mathrm{P}$, Heliövaara $\mathrm{M}$, Impivaara $\mathrm{O}$, et al. Long-term determinants of muscle strength decline: prospective evidence from the 22-year mini-Finland follow-up survey. J Am Geriatr Soc. 2012;60(1):77-85.

33. Avorn J, Langer E. Induced disability in nursing-home patients: a controlled trial. J Am Geriatr Soc. 1982;30(6):397-400.

34. Williams P, Lord SR. Effects of group exercise on cognitive functioning and mood in older women. Aust N Z J Public Health 1997;21(1):45-52.

35. Hulens M, Vasant G, Lysens R, Claessens AL, Muls E, Brumagne S. Study of differences in peripheral muscle strength of lean versus obese women: an allometric approach. Int J Obes Relat Metab Disord. 2001;25(5):67681.

36. Jürimäe $\mathrm{T}$, Jürimäe J. Anthropometric and health-related fitness characteristics in middle-aged obese women. Coll Antropol. 1998;22(1):97-106.

\section{Endereço para correspondência}

Thais de Lima Resende

Av. Ipiranga 6681 , prédio $12 \mathrm{~A}, 8^{\circ}$ andar

Porto Alegre/RS - CEP 90619-900

Telefone: +55 5133203646

E-mail: thaislr@pucrs.br 